\title{
Chlorinated hydrocarbons in beef, cow liver and milk products and total mercury in cow liver
}

\author{
Hilkka Tyllinen, Eeva Karppanen and Esko Nurmi \\ State Veterinary Medical Institute, 00550 Helsinki 55, Finland
}

\begin{abstract}
The chlorinated hydrocarbons used in pesticides and PCB-compounds (polychlorinated biphenyls) were determined in domestic and imported beef (79 samples), cow liver (195 samples), butter (112 samples), cheese (93 samples) and milk powder (58 samples); mercury was determined in cow liver (163 samples). The domestic samples (320 samples in total) were collected from different parts of Finland. The foreign samples (270 samples in total) came mainly from the Nordic countries and Central Europe.

The chlorinated hydrocarbons were determined by gas chromatography with an E.C. detector. The mercury determinations were made from freezedried samples using a Coleman Analyzer.

In the domestic samples the contents of chlorinated hydrocarbons were low and clearly below the FAO/WHO recommended limits. The pesticide quantities in the foreign samples were often higher, and in two cases the total DDT limit recommended by $\mathrm{FAO} / \mathrm{WHO}$ was exceeded. The mercury contents of the livers were low and of the same magnitude in all samples examined.
\end{abstract}

\section{Introduction}

The use of pesticides has been limited by law in Finland; the use of mercury has been controlled since the middle of the 1960's and that of chlorinated hydrocarbons since the beginning of the 1970's. However, since the decomposition of many organic compounds in nature is slow, they may accumulate in animals and through them enter into food. A corresponding tendency has been observed concerning many metals which in sufficiently high concentrations in food may be detrimental to human health. In addition to domestic products, fairly large quantities of imported foodstuffs are used, and our knowledge of their contents of harmful compounds is limited. Thus continued research and control in this field is required.

This study deals with domestic and imported beef and cow liver, which is an important imported raw material for the meat industry. As a second group, milk products (butter, cheese and milk powder), which have a large domestic consumption, have been chosen; in addition they are important export articles. Analyses of chlorinated hydrocarbons, including polychlorinated biphenyls (PCB) were taken from all samples. Total mercury determinations were made in cow liver. 


\section{Material}

Domestic beef and cow liver were obtained from five different slaughterhouses in Southern and Central Finland. Foreign beef and liver samples were obtained in connection with customs clearance of food lots coming from different countries (Table 1).

Butter, cheese and milk powder samples were obtained from dairies operating in different parts of Finland. Foreign milk products were random purchases mainly from other Nordic countries where similar studies have been made. The rest of the material consisted of occasional samples from Central and Southern Europe and the U.S.A. (Table 1).

Table 1. The samples from the various countries.

\begin{tabular}{|c|c|c|c|c|c|}
\hline Country & Beef & Cow liver & Butter & Cheese & Milk powder \\
\hline Australia ........................ & - & 21 & - & - & - \\
\hline Canada ............................ & - & 10 & - & - & - \\
\hline Czechoslovakia .............. & - & - & 1 & - & - \\
\hline Denmark ......................... & - & - & 13 & 7 & - \\
\hline Finland ............................ & 64 & 55 & 73 & 75 & 53 \\
\hline France ........................... & - & - & 2 & 9 & - \\
\hline Ireland ….......................... & 5 & 17 & 3 & 1 & - \\
\hline Netherlands ................... & - & - & 1 & - & - \\
\hline New Zealand ................. & - & 19 & - & - & - \\
\hline 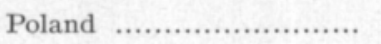 & 10 & 52 & - & - & - \\
\hline Spain & - & - & 2 & - & - \\
\hline Sweden & - & 12 & 9 & - & 3 \\
\hline Switzerland .................... & - & - & 1 & - & 1 \\
\hline U.K. ........................ & - & - & 2 & - & - \\
\hline 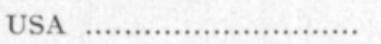 & - & 9 & 3 & - & 1 \\
\hline West Germany ................ & - & - & 2 & 1 & - \\
\hline Number of samples ......... & 79 & 195 & 112 & 93 & 58 \\
\hline
\end{tabular}

\section{Methods}

Chlorinated hydrocarbons

Extraction and cleanup

The fresh sample (beef 10 grams, cow liver 5 grams, butter 1 gram, cheese $2-10$ grams, milk powder $4-50$ grams) was homogenized with anhydrous sodium sulphate. The homogenate was then shaken in a flask with a glass plug with diethyl ether $(25$ to $100 \mathrm{ml}$ ) about 3 minutes and the extract was filtered through glass wool. The ether was evaporated at room temperature. The fat was dissolved in $5 \mathrm{ml}$ of hexane and decanted into a separatory funnel $(250 \mathrm{ml})$ and rinsed with $3 \times 3 \mathrm{ml}$ of hexane. Fifteen milliliters of acetonitrile saturated with hexane were added and shaken vigorously for $1-2$ minutes. After the layers had separated the acetonitrile was drained into a $150 \mathrm{ml}$ glass bowl. The shaking was repeated with three $15 \mathrm{ml}$ portions of acetonitrile. The acetonitrile extract was evaporated at $60-70^{\circ} \mathrm{C}$ by blowing purified nitrogen slowly down into the glass bowl. Methylene chloride was added in a sufficient 
amount and the fat in the methylene chloride solution was then applied on $\mathrm{e}$ thin layer plate.

The thin layer plates $(50 \times 200 \mathrm{~mm})$ were covered with Silica Gel G so that the layer was $1 \mathrm{~mm}$ thick (Shandon Equipment for Thinlayer Chromatography) (KARPPANEN et al. 1969). The solvent fronts, the border line between the upper and lower section and the origin line were marked on the plates, which were then heated overnight at $200^{\circ} \mathrm{C}$. The plates with fat on the origin line were developed with the solvent methylene chloride and n-hexane, 25: $75(\mathrm{v} / \mathrm{v})$. After drying at $25^{\circ} \mathrm{C}$ both the upper and the lower sections of the thin layer material were removed and packed into small glass columns. They were then extracted with cyclohexane and diethyl ether, 6:4 (v/v). The eluates were collected in $1 \mathrm{ml}$ flasks. The solution from the upper section contained PCB compounds, p,p'DDE and p,p'DDT. To separate p,p'DDT and a major PCB peak $0.5 \mathrm{ml}$ of the eluate was treated with a methanol solution of 10 per cent $\mathrm{KOH}(\mathrm{w} / \mathrm{w})$.

\section{Gas chromatography}

Gas chromatograph: Varian 600 and 1400 with electron capture detector (tritium) and glass columns.

Column packing:

1) Chromosorb W a.w./DMCS. 100/120 mesh; coated with $5 \%$ SF96 methyl silicone.

2) As above but stationary phase $5 \%$ SF96-QF-1, 25: 75.

Carrier gas: Nitrogen $99.999 \%$.

Reference solutions: Pesticides, purity 99-100\% (Analytical Standards LTD, Sweden).

Reference solutions of PCB:

1) A hexane solution of a $60 \%$-chlorinated technical PCB-micture (Clophen A 60).

2) A hexane solution of a $42 \%$-chlorinated technical PCB-mixture (Aroclor 1242).

\section{Calculation of PCB}

After the pesticide residues from the sample had been removed, the sum of the heights of all peaks corresponding to PCB peaks was calculated with the exception of the two interfering with p.p'DDE. This sum was compared with the sum obtained from the same peaks in the reference chromatograms.

By using the method of acetonitrile partition (ZWEIG and SHERMA 1972) it has been shown that both pesticides and PCB decrease. Average recovery of pesticides is $95 \%$ and that of $\mathrm{PCB} 70 \%$. Organochlorine compounds may decrease during evaporation of acetonitrile.

\section{Total mercury}

For determination of total mercury the samples were analysed by an oxygen flask combustion method (Gutenmann and Lisk 1960, RAJAma et al. 1964) combined with a Mercury Analyzer Coleman 50 (Perkin-Elmer). 
Table 2. Organo chlorine content and total mercury in cow liver. Mean concentration, mg/ kg fresh sample. (Range in parentheses)

\begin{tabular}{|c|c|c|c|c|c|c|}
\hline Country & Number & Dieldrin & Total DDT & PCB & Fat- $\%$ & $\mathrm{Hg}$ \\
\hline \multicolumn{7}{|l|}{ Australia } \\
\hline $1971 \ldots \ldots \ldots \ldots \ldots$ & 13 & $-1)$ & $\begin{array}{c}0.007 \\
\text { (n.d. }- \\
0.024)\end{array}$ & $\begin{array}{c}0.008 \\
(<0.005- \\
0.021)\end{array}$ & $\begin{array}{c}3,5 \\
(2,4-6,0)\end{array}$ & $\begin{array}{c}0,06 \\
\text { (n.d. }-0.12 \text { ) }\end{array}$ \\
\hline $1972 \ldots \ldots \ldots \ldots \ldots$ & 3 & n.d. & $\begin{array}{l}<0.005 \\
(<0.005- \\
<0.005)\end{array}$ & $\begin{array}{l}<0.005 \\
(<0.005- \\
<0.005)\end{array}$ & $(2.6-3.6)$ & $\begin{array}{c}0.05 \\
(0.03-0.07)\end{array}$ \\
\hline $1973 \ldots \ldots \ldots \ldots \ldots$ & 5 & n.d. & $\begin{array}{l}<0.005 \\
(<0.005- \\
<0.005)\end{array}$ & $\begin{array}{l}<0.005 \\
(<0.005- \\
<0.005)\end{array}$ & $(3.6-5.5)$ & $\begin{array}{c}0.04 \\
(0.02-0.05)\end{array}$ \\
\hline \multicolumn{7}{|l|}{ Canada } \\
\hline $1974 \ldots \ldots \ldots \ldots \ldots$ & 10 & n.d. & $\begin{array}{c}<0.005 \\
\text { (n.d. }-\overline{0} \\
0.010)\end{array}$ & $\begin{array}{c}0.006 \\
(<0.005- \\
0.027)\end{array}$ & $(2.6-5.8)$ & $-2)$ \\
\hline \multicolumn{7}{|l|}{ Finland } \\
\hline & & & $\begin{array}{c}\text { (n.d.- } \\
0.006)\end{array}$ & $\begin{array}{c}(<0.005- \\
0.079\end{array}$ & $(1.5-4.1)$ & (n.d. -0.09 ) \\
\hline $1972 \ldots \ldots \ldots \ldots \ldots$ & 27 & n.d. & $\begin{array}{l}<0.005 \\
\text { (n.d. }- \\
<0.005 \text { ) }\end{array}$ & $\begin{array}{l}<0.005 \\
\text { (n.d. }- \\
<0.005 \text { ) }\end{array}$ & $\begin{array}{c}3.3 \\
(2.0-9.1)\end{array}$ & $\begin{array}{c}0.06 \\
\text { (n.d. }-0.12 \text { ) }\end{array}$ \\
\hline $1974 \ldots \ldots \ldots \ldots \ldots$ & 12 & n.d. & $\begin{array}{l}<0.005 \\
\text { (n.d. }- \\
<0.005)\end{array}$ & $\begin{array}{c}0.010 \\
(<0.005- \\
0.025)\end{array}$ & $\begin{array}{c}3.2 \\
(2.5-4.1)\end{array}$ & $-2)$ \\
\hline $\begin{array}{l}\text { Ireland } \\
1972 \ldots \ldots \ldots \ldots \ldots \ldots\end{array}$ & 17 & n.d. & $<0.005$ & $<0.005$ & 2.8 & 0.05 \\
\hline N 7 Th & & & $\begin{array}{c}(<0.005- \\
<0.005)\end{array}$ & $\begin{array}{c}(<0.005- \\
<0.005)\end{array}$ & $(2.3-4.0)$ & (n.d. -0.12 ) \\
\hline \multicolumn{7}{|l|}{ New Zealand } \\
\hline & 19 & & (n.d. $-\overline{0}$ & $\begin{array}{l}(0.005- \\
0.043)\end{array}$ & $(2.0-6.4)$ & (n.d. -0.04 ) \\
\hline \multicolumn{7}{|l|}{ Poland } \\
\hline 1971 ................ & 8 & $-1)$ & $\begin{array}{c}0.025 \\
(0.012- \\
0.040)\end{array}$ & $\begin{array}{c}0.012 \\
(0.005- \\
0.055)\end{array}$ & $\begin{array}{c}3.1 \\
(2.1-7.1)\end{array}$ & $\begin{array}{c}0.08 \\
(0.04-0.13)\end{array}$ \\
\hline 1972 .............. & 44 & $\begin{array}{l}<0.005 \\
\text { (n.d. }- \\
<0.005 \text { ) }\end{array}$ & $\begin{array}{c}0.035 \\
(<0.005- \\
0.39)\end{array}$ & $\begin{array}{c}0.006 \\
\text { (n.d. }- \\
0.086)\end{array}$ & $(1.6-10.5)$ & $\begin{array}{c}0.07 \\
(0.03-0.11)\end{array}$ \\
\hline $\begin{array}{l}\text { Sweden } \\
1972 ~ \ldots \ldots \ldots \ldots \ldots \ldots . . . . .\end{array}$ & 2 & n.d. & $\begin{array}{l}<0.005 \\
(<0.005- \\
<0.005)\end{array}$ & $\begin{array}{l}<0.005 \\
(<0.005- \\
<0.005)\end{array}$ & $\begin{array}{c}2.9 \\
(2.7-3.2)\end{array}$ & $\begin{array}{c}0.10 \\
(0.08-0.11)\end{array}$ \\
\hline $1973 \ldots \ldots \ldots \ldots \ldots$ & 10 & n.d. & $\begin{array}{c}0.006 \\
(<0.005- \\
0.030)\end{array}$ & $\begin{array}{l}0.051 \\
\left(\begin{array}{l}0.029- \\
0.077\end{array}\right)\end{array}$ & $\begin{array}{c}3.1 \\
(2.4-4.7)\end{array}$ & n.a. \\
\hline $\begin{array}{l}\text { USA } \\
1971 \ldots \ldots \ldots \ldots \ldots \ldots\end{array}$ & 9 & $-1)$ & $\begin{array}{c}0.011 \\
\text { (n.d. }- \\
0.024)\end{array}$ & $\begin{array}{c}<0.005 \\
(<0.005- \\
0.010)\end{array}$ & $\begin{array}{c}3.2 \\
(2.3-6.8)\end{array}$ & $\begin{array}{c}0.13 \\
\text { (n.d. }-0.37)\end{array}$ \\
\hline $\begin{array}{l}\text { Number of } \\
\text { samples ........... }\end{array}$ & 195 & & & & & \\
\hline
\end{tabular}

samples ............ 195

1) $\mathrm{H}_{2} \mathrm{SO}_{4}$-method.

2) STABEL-TAUCHER et al. 1975.

n.d. $=$ not detected n.a. $=$ not analysed 


\section{Results and discussion}

In the result tables only those chlorinated hydrocarbons detected in the samples are shown. The sum of DDT compounds was calculated and mentioned in the tables as total DDT.

Dieldrin was found in small quantities only in the Polish liver samples (Table 2). However, parts of the liver samples have been cleaned by the $\mathrm{H}_{2} \mathrm{SO}_{4}$-method (AHLING and JENSEN 1970), during which the dieldrin dissolves and cannot be detected. The highest total DDT value was also detected in Polish liver. If the results are compared to the suggested FAO/WHO limit of $7 \mathrm{mg} / \mathrm{kg}$ fat (ANON. 1973), one Polish liver sample exceeded this value. In the domestic liver samples the total DDT content was low. In one of the domestic samples an abnormally high PCB content was found. The sample was from a cow which during the grazing period had been drinking water from the Kokemäki river; this river belongs to the Finnish water system most seriously polluted by PCB compounds. The mercury contents of the livers were low and of the same magnitude in the different countries. The highest values were found in liver imported from the U.S.A., but the limited material does not justify definite conclusions.

In the Finnish and Irish beef samples examined, only small residues of DDT decomposition products were found (Table 3). On the other hand, the average total DDT in the Polish beef samples examined was considerably higher, exceeding in one sample the $\mathrm{FAO} / \mathrm{WHO}$ recommended limit of $7 \mathrm{mg} /$ $\mathrm{kg}$ fat (Anon. 1973). Small amounts of PCB were found in Finnish beef samples only.

In the Finnish, Swedish, Danish and Irish butter samples analysed, the contents of chlorinated hydrocarbons were of the same magnitude and fairly low (Table 4). Results in agreement with this study have also been obtained in Sweden (Westöö et al. 1970), Denmark (Bro-RAsmussen et al. 1968) and Ireland (Downey 1972). The Danes, however, have recorded considerable BHC-values ( $\beta$-BHC and lindane), whereas the 13 Danish butter samples in

Table 3. Organo chlorine content in beef. Mean concentration, $\mathrm{mg} / \mathbf{k g}$ fresh sample. (Range in parentheses).

\begin{tabular}{|c|c|c|c|c|}
\hline Country & Number & Total DDT & РCB & Fat $\%$ \\
\hline Finland 1974 ............... & 64 & $\begin{array}{c}<0.005 \\
\text { (n.d. }-<0.005)\end{array}$ & $\begin{array}{c}<0.005 \\
(<0.005-<0.005)\end{array}$ & $\begin{array}{c}5.0 \\
(0.9-14.9)\end{array}$ \\
\hline Ireland $1973 \ldots \ldots \ldots \ldots$ & 5 & $\begin{array}{c}<0.005 \\
\text { (n.d. }-<0.005)\end{array}$ & n.d. & $\begin{array}{c}23.8 \\
(17.3-30.2)\end{array}$ \\
\hline Poland 1973 .............. & 10 & $\begin{array}{r}0.69 \\
(0.052-4.4)\end{array}$ & n.d. & $\begin{array}{c}23.7 \\
(12.5-34.1)\end{array}$ \\
\hline Number of samples ... & 79 & & & \\
\hline
\end{tabular}

n.d. $=$ not detected 
Table 4. Organo chlorine content in butter. Mean concentration, $\mathrm{mg} / \mathrm{kg}$. Range in parentheses.

\begin{tabular}{|c|c|c|c|c|c|c|}
\hline Country & Number & Lindane & $\begin{array}{l}\text { Heptachlor- } \\
\text { epoxide }\end{array}$ & Dieldrin & Total DDT & PCB \\
\hline \multicolumn{7}{|l|}{ Denmark } \\
\hline $1972 \ldots \ldots \ldots \ldots \ldots$ & 4 & n.d. & n.d. & $\begin{array}{c}0.011 \\
(0.009- \\
0.012)\end{array}$ & $\begin{array}{c}0.016 \\
(0.007- \\
0.022)\end{array}$ & $\begin{array}{l}0.15 \\
(0.063- \\
0.24)\end{array}$ \\
\hline $1973 \ldots \ldots \ldots \ldots \ldots$ & 9 & n.d. & n.d. & $\begin{array}{c}0.006 \\
(<0.005- \\
0.011)\end{array}$ & $\begin{array}{c}0.017 \\
(0.009- \\
0.034)\end{array}$ & $\begin{array}{c}0.21 \\
(0.090- \\
0.35)\end{array}$ \\
\hline \multicolumn{7}{|l|}{ Finland } \\
\hline $1972 \ldots \ldots \ldots \ldots \ldots$ & 42 & n.d. & n.d. & $\begin{array}{r}<0.005 \\
\text { (n.d. - } \\
0.006)\end{array}$ & $\begin{array}{c}0.011 \\
(<0.005- \\
0.022)\end{array}$ & $\begin{array}{c}0.065 \\
(0.028- \\
0.18)\end{array}$ \\
\hline $1973-1974 \ldots \ldots$ & 31 & n.d. & n.d. & n.d. & $\begin{array}{c}<0.005 \\
(<0.005- \\
0.006)\end{array}$ & $\begin{array}{c}0.076 \\
(0.028- \\
0.14)\end{array}$ \\
\hline \multicolumn{7}{|l|}{ Ireland } \\
\hline $1973 \ldots \ldots \ldots \ldots \ldots$ & 3 & $\begin{array}{c}<0.005 \\
(<0.005- \\
<0.005)\end{array}$ & n.d. & $\begin{array}{c}0.013 \\
(0.011- \\
0.017)\end{array}$ & $\begin{array}{c}0.012 \\
(0.095- \\
0.15)\end{array}$ & $\begin{array}{c}0.090 \\
(0.046- \\
0.14)\end{array}$ \\
\hline \multicolumn{7}{|l|}{ Sweden } \\
\hline $1972 \ldots \ldots \ldots \ldots \ldots$ & 7 & n.d. & n.d. & $\begin{array}{r}<0.005 \\
(\mathrm{n} . \mathrm{d} .- \\
0.012)\end{array}$ & $\begin{array}{c}0.034 \\
(0.010- \\
0.065)\end{array}$ & $\begin{array}{c}0.094 \\
(0.075- \\
0.12)\end{array}$ \\
\hline $1973 \ldots \ldots \ldots \ldots \ldots$ & 2 & n.d. & n.d. & $\begin{array}{c}0.011 \\
(0.007- \\
0.016)\end{array}$ & $\begin{array}{r}<0.005 \\
\quad \text { (n.d. }- \\
<0.005 \text { ) }\end{array}$ & $\begin{array}{c}0.11 \\
(0.086- \\
0.13)\end{array}$ \\
\hline \multicolumn{7}{|l|}{$\begin{array}{l}\text { The other } \\
\text { countries }\end{array}$} \\
\hline $1972 \ldots \ldots \ldots \ldots \ldots$ & 14 & n.d. & $\begin{array}{l}<0.005 \\
\text { (n.d. }- \\
<0.005 \text { ) }\end{array}$ & $\begin{array}{c}0.021 \\
(<0.005- \\
0.050)\end{array}$ & $\begin{array}{c}0.051 \\
(<0.005- \\
0.27)\end{array}$ & $\begin{array}{c}0.090 \\
(0.035- \\
0.24)\end{array}$ \\
\hline $\begin{array}{l}\text { Number of } \\
\text { samples ............ }\end{array}$ & 112 & & & & & \\
\hline
\end{tabular}

n.d. $=$ not detected.

our material did not contain detectable amounts of these compounds. This may depend on differences in the methodology.

There were no significant differences in the average residue contents of the Finnish and Danish cheese samples (Table 5). However, there was in the Danish samples some heptachlorepoxide, which was not found in the Finnish samples. In the cheese samples originating from other countries (France, Germany) clearly higher amounts of dieldrin and DDT were found than in samples from the Nordic countries.

All the examined milk powder samples contained only small residues of chlorinated hydrocarbons (Table 6). 
Table 5. Organo chlorine content in cheese. Mean concentration, $\mathrm{mg} / \mathrm{kg}$ fresh sample. (Range in parentheses).

\begin{tabular}{lccccc}
\hline Country Number Lindane & $\begin{array}{c}\text { Heptachlor- } \\
\text { epoxide }\end{array}$ & Dieldrin & Total DDT PCB \\
\hline
\end{tabular}

\section{Denmark}

\begin{tabular}{|c|c|c|c|c|c|c|}
\hline $1972 \quad \ldots \ldots \ldots \ldots \ldots$ & 5 & n.d. & $\begin{array}{l}<0.005 \\
\text { (n.d. }- \\
<0.005 \text { ) }\end{array}$ & $\begin{array}{r}<0.005 \\
\quad(\text { n.d. }- \\
<0.005)\end{array}$ & $\begin{array}{c}0.005 \\
(<0.005- \\
0.008)\end{array}$ & $\begin{array}{c}0.058 \\
(0.042- \\
0.10)\end{array}$ \\
\hline $1973 \ldots \ldots \ldots \ldots \ldots$ & 2 & n.d. & n.d. & $\begin{array}{c}<0.005 \\
(<0.005- \\
<0.005)\end{array}$ & $\begin{array}{c}<0.005 \\
(<0.005- \\
<0.005)\end{array}$ & $\begin{array}{c}0.090 \\
(0.085- \\
0.094)\end{array}$ \\
\hline \multicolumn{7}{|l|}{ Finland } \\
\hline 1972 ................ & 26 & n.d. & n.d. & $\begin{array}{r}<0.005 \\
\quad(\text { n.d. }- \\
<0.005)\end{array}$ & $\begin{array}{c}<0.005 \\
(<0.005- \\
0.007)\end{array}$ & $\begin{array}{c}0.068 \\
(0.008- \\
0.19)\end{array}$ \\
\hline 1973 ................ & 49 & n.d. & n.d. & $\begin{array}{r}<0.005 \\
\quad(\text { n.d. }- \\
<0.005)\end{array}$ & $\begin{array}{r}<0.005 \\
\text { (n.d.- } \\
0.005 \text { ) }\end{array}$ & $\begin{array}{c}0.074 \\
(0.007- \\
0.35)\end{array}$ \\
\hline \multicolumn{7}{|l|}{$\begin{array}{l}\text { The other } \\
\text { countries }\end{array}$} \\
\hline 1972 ................. & 7 & n.d. & $\begin{array}{c}0.020 \\
\text { (n.d. }-0.12 \text { ) }\end{array}$ & $\begin{array}{r}0.020 \\
\text { (n.d. - } \\
0.086)\end{array}$ & $\begin{array}{c}0.018 \\
(<0.005- \\
0.059)\end{array}$ & $\begin{array}{c}0.10 \\
(0.069- \\
0.17)\end{array}$ \\
\hline $1973 \ldots \ldots \ldots \ldots . . . . . .$. & 4 & $\begin{array}{c}0.014 \\
(<0.005- \\
0.022)\end{array}$ & n.d. & $\begin{array}{c}0.008 \\
(<0.005- \\
0.015)\end{array}$ & $\begin{array}{c}0.016 \\
(<0.005- \\
0.031)\end{array}$ & $\begin{array}{c}0.10 \\
(0.023- \\
0.17)\end{array}$ \\
\hline $\begin{array}{l}\text { Number of } \\
\text { samples ............ }\end{array}$ & 93 & & & & & \\
\hline
\end{tabular}

n.d. = not detected.

Table 6. Organo chlorine content in milk powder. Mean concentration, mg/kg. (Range in parentheses).

\begin{tabular}{|c|c|c|c|c|}
\hline Country & Number & Dieldrin & Total DDT & PCB \\
\hline Finland 1972 ............... & 14 & n.d. & $\begin{array}{r}<0.005 \\
(\text { n.d. }-0.010)\end{array}$ & $\begin{array}{c}0.017 \\
(<0.005-0.054)\end{array}$ \\
\hline 1973 ........................... & 39 & $\begin{array}{c}<0.005 \\
\text { (n.d. }-<0.005)\end{array}$ & $\begin{array}{c}<0.005 \\
(\text { n.d. }-<0.005)\end{array}$ & $\begin{array}{c}0.005 \\
(<0.005-0.021)\end{array}$ \\
\hline Sweden 1972 ............. & 3 & n.d. & $\begin{array}{c}<0.005 \\
(\text { n.d. }-<0.005)\end{array}$ & $\begin{array}{c}0.005 \\
(<0.005-0.007)\end{array}$ \\
\hline Switzerland $1972 \ldots \ldots \ldots$ & 1 & $<0.005$ & $<0.005$ & 0.005 \\
\hline USA 1972 ................. & 1 & $<0.005$ & $<0.005$ & 0.022 \\
\hline Number of samples ... & 58 & & & \\
\hline
\end{tabular}


Ahling, B. \& Jensen, S. 1970. Anal. Chem. 42: 1483.

Anon. 1973. Pesticide residues in food. Report of the 1972 Joint FAO/WHO Meeting. WHO Techn. Rep. Ser. 1973: 525.

Bro-Rasmussen, F., Dalgaar-Mikkelsen, SV., Jakobsen, TH., Koch, SV. O., Rodin, F., Uht, E. \& Voldum-Clausen, K. 1968. Examinations of Danish milk and butter for contaminating organochlorine insecticides. Residue Rev. 3: 55-69.

Downey, W. K. 1972. Pesticide residues in milk and milk products. Ann. Bull. 2.

Gutenmann, W. H. \& Lisk, D. J. 1960. Rapid determination of mercury in apples by modified Schöninger combustion. Agric. Food Chem. 8:306-308.

Karppanen, E., Henriksson, K. \& Helminen, M. 1969. OECD Study of Pesticide Residues 1967-1968. Finland Report. Joint OECD/TNO Meeting at „De Guldenberg Helvoirt. The Netherlands. September 15-18.

Rajama, J., Hiltunen, S. \& Hilpi, A. 1964. An oxygenflask combustion method for the determination of mercury in eggs. Rep. Chem. 4.

Stabel-Taucher, R. Nurmi, E. \& Karppanen, E. 1975. Content of copper, zinc, lead, cadmium and mercury in muscle, liver and kidney of Finnish cattle. J. Scient. Agric. Soc. Finl. 47:000-000.

Westöö, G., Norén, K. \& Andersson, M. 1970. Levels of organochlorine pesticides and polychlorinated biphenyls in margarine, vegetable oils and some foods of animal origin on the Swedish market in 1967-1969. Vår Föda 2-3:10-31.

Zweig, G. \& Sherma, J. 1972. Chlorinated pesticides. Analytical Methods for Pesticides and Plant Growth Regulators 6:132-134. New York.

\title{
SELOSTUS
}

\section{Klooratut hiilivedyt naudan lihassa, naudan maksassa ja maitotuotteissa sekä kokonaiselohopea naudan maksassa}

\author{
Hilkka Tyllinen, Eeva Karppanen ja Esko Nurmi \\ Valtion eläinlääketieteellinen laitos, 00550 Helsinki 55
}

Torjunta-aineiden käyttöä on rajoitettu ja käytön valvontaa tehostettu Suomessa kloorattujen hiilivetyjen osalta 1970-luvun alusta alkaen ja elohopean osalta jo 1960-luvun puolivälistä lähtien. Useiden orgaanisten yhdisteiden hajoaminen luonnossa on kuitenkin hyvin hidasta, joten niillä samoin kuin monilla metalleilla on mahdollisuus rikastua erikoisesti eläinkudoksiin ja sitä kautta elintarvikkeisiin. Kotimaisten tuotteiden ohella käytetään maassamme runsaasti ulkomaisia elintarvikkeita. Tiedot näiden sisältämistä haitallisista yhdisteistä ovat vähäiset.

Tähån tutkimukseen on valittu sekä kotimainen että ulkomailta tuotu naudan liha, josta on määritetty tuhoeläinmyrkkyinä käytetyt klooratut hiilivedyt ja PCB-yhdisteet 79 näytteestä. Naudan maksasta, joka on tärkeä lihateollisuuden raaka-aineeksi käytetty tuontituote, on vastaavia määrityksiä suoritettu 195 näytteestä. Toiseksi ryhmäksi on otettu maitotuotteet, joilla on suuri kotimainen kulutus, ja lisäksi ne ovat tärkeitä vientiartikkeleita. Voinäytteitä on analysoitu 112, juustonäytteitä 93 ja maitojauhenäytteitä 58. Vuosina 1971-1973 on suoritettu kokonaiselohopeamääritykset 163 naudan maksanäytteistä, ja nämä tulokset on liitetty tähän tutkimukseen. Eri puolilta Suomea kerättyjä kotimaisia näytteitä oli kaikkiaan 320. Ulkomaisia näytteitä oli yhteensä 217 ja ne olivat etupäässä Pohjoismaista ja Keski-Euroopasta.

Kotimaisissa näytteissä torjunta-aineina käytettyjen kloorattujen hiilivetyjen pitoisuudet olivat alhaiset ja alittivat selvästi FAO/WHO:n suosittelemat raja-arvot. Ulkomaisten näytteiden pestisidimäärät vaihtelivat suuresti. Joissakin maissa ne olivat samaa suuruusluokkaa kuin Suomessa, toisissa taas huomattavasti korkeampia. Kahdessa tapauksessa FAO/WHO:n kokonais-DDT:lle suosittelema raja-arvo ylitettiin. PCB-yhdisteiden jäämät olivat yleensä pieniä ja vaihtelivat samoissa rajoissa sekä kotimaisissa että ulkomaisissa näytteissä. Maksojen elohopeapitoisuudet olivat kaikissa tutkituissa näytteissä alhaisia ja suuruusluokaltaan samanlaisia. 